\title{
Staying neutral or intervening?
}

\section{Ethics teachers' ideas on how to respond to alarming cases brought forward by medical students in class: A qualitative study in the Netherlands}

\author{
Maartje Hoogsteyns $^{1}$ (D) Amalia Muhaimin ${ }^{1,2}$
}

Accepted: 21 April 2021 / Published online: 3 June 2021

(C) The Author(s) 2021

\begin{abstract}
Ethics teachers are regularly confronted with disturbing cases brought in by medical students in class. These classes are considered confidential, so that everyone can speak freely about their experiences. But what should ethics teachers do when they hear about a situation they consider to be outright alarming, for example where patients/students' safety is at stake or where systematic power abuse seems to be at hand? Should they remain neutral or should they step in and intervene? In the Netherlands, as in many other countries, there are no clear guidelines for ethics teachers on how to respond. To get more insight into what teachers themselves think a proper response would be, we interviewed 18 Dutch medical ethics teachers. We found that Dutch ethics teachers will address the issue in class, but that they are overall reluctant to intervene; take action outside the scope of class. This reluctance is partly rooted in the conviction that ethicists should stay neutral and facilitate reflection, instead of telling students or physicians what to do. At the same time, this neutral position seems a difficult place to leave for those teachers who would want to or feel they need to. This has to do with various organizational and institutional constraints tied up with their position. The study invites medical ethics teachers to reflect on these constraints together and think about how to proceed from there. This study seeks to contribute to research on cultural change in medicine and medical students' experiences of moral distress.
\end{abstract}

Keywords Moral distress · Medical students · Ethics teachers · Responses · Alarming cases · Qualitative research

Maartje Hoogsteyns

m.hoogsteyns@amsterdamumc.nl

Extended author information available on the last page of the article 


\section{Introduction}

Medical ethics teachers are regularly confronted with disturbing cases brought in by medical students. Especially in the master phase, when interns are asked to share personally observed ethical issues, difficult dilemmas can come by. In small-group ethics classes students learn to morally reflect together and because the cases are grounded in personal experience learning is suggested to take place on a deeper level (Kolb 2014). These meetings are confidential, so that everyone can speak freely about their thoughts and share experiences.

But what should ethics teachers do when they hear about a situation that they consider to be outright alarming, for example where patients/students' safety is at stake or where systematic power abuse seems to be at hand? Should they stay neutral and consider it part of students' learning trajectories? Or are they, in fact, obliged to report the case? Should they guard confidentiality or should they be able to at least discuss it with colleagues?

In the Netherlands, physicians and nurses have a duty to report incidents (mistakes or unforeseen events in care situations) or calamities (incidents that are harmful for patients), for example through VIM (Veilig Incidenten Melding- Safe Reporting of Incidents), so that health care organizations can learn from them. This can be done anonymously. For medical psychologists and hospital counselors confidentiality regulations are in place. But there seem to be no clear guidelines for ethics teachers on how to respond to an alarming case.

This is not necessarily a bad thing; teachers might appreciate the possibilities to react to such situation with contextual sensitivity. But absence of clarity could also undermine the willingness to get involved in addressing an alarming case in the first place. Henriksen and Dayton (2006) did research on organizational silence and hidden threats to patient safety and write that when individuals are in doubt or not so secure about their roles, they are less "likely to transcend individual concerns and speak up regarding higher-order organizational concerns".

So to get more insight into how ethics teachers perceive their own role in the academic hospital-setting, we wanted to know what teachers themselves think a proper response would look like. We interviewed 18 Dutch medical ethics teachers and asked them if they ever met with cases from interns they felt were alarming, how they responded to them, or think they should have responded, and what reasons they had for doing so. By gathering teachers' experiences and views, we hoped to learn where ethics teachers feel their responsibility for the well-being of students (and patients) begins and ends, how they think they can support students in such a situation, and how they themselves perhaps can be supported in dealing with this type of information.

So where do we draw the boundary between a disturbing and an alarming case? In our study, we constructed the following definition: a case is alarming when the situation described is considered to be so harmful (for a patient, patients' family, student, colleague) or unjust that it appeals to the teacher's personal sense of morality. An alarming case evokes a feeling of disbelief, worry or even outrage in the teacher about the situation described ('I cannot believe I am hearing this!!', 'But something should have been done!'). Such cases make the teacher doubt as how to respond properly - instead of just treating the case as any other. Of course, this experience will vary between teachers and will also change during their teaching career. 


\section{Methodology}

Six out of eight Dutch academic hospitals offer small-scale classes in which students discuss personally observed ethical cases during the master phase. We limited our study to these six academic hospitals and made sure to interview at least two teachers in each hospital. We mainly spoke to regular staff (assistant and associate professors), because they teach such classes more regularly. But we also talked to three teaching PHDstudents.

Our goal was to explore the variety of perspectives and experiences present among ethics teachers, so we made a purposive sample. The variety between participants was wide in terms of age (between 22 and 65 years), gender and educational background. About half of the teachers had finished a study in medicine or nursing, before turning to a study in ethics or law. Two of them were practicing physicians (pediatrician and GP) at the moment of the interview, three had worked earlier as a nurse. The other teachers were trained in philosophy, humanity, psychology or pedagogics. Two of them had experience working as spiritual caregiver.

Participants were approached through email. 18 out of 20 teachers who actually were (or had been) involved in discussing cases from medical students were willing to participate. We obtained permission to have the interview audio recorded and agreed that all data were kept anonymous and unidentifiable to ensure the teachers' privacy and confidentiality.

The semi-structured interviews were held in the working environment of the interviewee and took between 45 and $90 \mathrm{~min}$. The first 14 interviews were conducted by $\mathrm{AM}$ and RBW, in English, the last four interviews were done by $\mathrm{MH}$ in Dutch. AM is a physician and ethics teachers in Indonesia, RBW is a physician and teacher (in Indonesia) and cooperated with AM during the interviews and later transcribed part of the interviews. $\mathrm{MH}$ is an ethics teacher in the Netherlands and is, like AM, trained in doing qualitative research. AM and $\mathrm{MH}$ both met with alarming cases in their ethics classes and could therefore easily relate to the issue discussed.

In the first 14 interviews, the questions about the alarming cases were part of a larger list of open questions on the goals and organization of ethics education in the clerkship phase, as part of a comparative study between Indonesia and the Netherlands (Muhaimin et al. 2019, 2020). Somewhere in the interview, the following question was posed: "Have you had any experience with hearing a case from a student that you thought was harmful for a patient or student, and that you thought you needed to do something about?" This part of the interview took 15 to 20 min on average. We decided to focus on cases that students share with ethics teachers in an educational setting and not to mix these up with cases that students brought to an ethics department on their own initiative, for example to address medical misconduct.

After reading and analyzing the transcripts by $\mathrm{AM}$ and $\mathrm{MH}$, four more interviews, focusing on this topic alone, were conducted by $\mathrm{MH}$ (that took 45 to $60 \mathrm{~min}$ ). In this way all Dutch academic hospitals were evenly represented in the study and it also gave the team a chance to check if any new views or arguments on how to respond would pop up, which was not the case. We had reached data saturation.

Because of the limited amount of interviews, we preferred to code and organize groups of codes manually (using excel sheets) instead of using a coding program. Analysis was done using a grounded theory approach and codes were regrouped 
Table 1 examples of alarming cases mentioned by interviewees

Interns are asked to conceal/be silent about medical errors in OR (to patient, family or colleagues)

Interns are asked to do consultations with patients without a physician present and/or are stimulated by seniors to pretend to patients they are already physicians

Interns witness a physician promoting his private clinic by commenting on the looks of patients Interns are asked to perform vaginal touché in OR without consent of the patient

Interns witnessing and having to take part in systematic breaking of confidentiality rules in relation to patient information

several times (axially) to see what themes would emerge. $\mathrm{MH}$ and $\mathrm{AM}$ discussed analyses to reach a consensus in the interpretation. Creating a separation between teachers' practical reasons (to respond in this way or another) and more ideological motivations (to respond this way or another) appeared helpful to organize the data in a meaningful way. After analysis, the results were member checked with four interviewees.

\section{Results}

16 out of 18 teachers had indeed come across cases from interns they considered alarming. All of them stressed that this did not happen regularly though; alarming cases are considered exceptions.

\section{Alarming cases}

To give an impression of the kind of cases teachers mentioned, we present some examples in Table 1 below.

The focus of teachers in these cases was mainly on the learning environment of the student. Several teachers, for example, talked about medical errors made in the OR. Teachers were not so much alarmed by the error itself, but by the fact that it was silenced and that there had been no opportunity for the team and students to learn from mistakes made.

" Doctors in the OR panicked and were blaming each other and interns were ordered to keep silent. An extreme bad example for students” (Interview 17)

Cases that seemed to re-occur at a certain department evoked a stronger sense of alarm than singular incidents. Except for the case of vaginal touché, all alarming cases were not so much considered dilemmatic by teachers, but straight-out wrong. ${ }^{1}$ Besides real

\footnotetext{
${ }^{1}$ Interns performing intimate examinations on patients in the OR, without the patient's consent. Some students and teachers were outraged by this 'common practice', others did not see real harm in it. On a national level the Royal Association of Medicine has clearly stated that this practice is not allowed. On a global level, this practice is regularly topic of research (see for example Rees and Monrouxe 2011).
} 
Table 2 Hypothetical alarming cases mentioned by interviewees

"When the quality of care is seriously impaired"

"When harm to patients can be prevented"

Systematic discrimination of students by doctors because of gender, sexual preference or ethnicity

Sexual relations between doctors and interns

cases, teachers also mentioned hypothetical cases or situations that would alarm them (see Table 2).

These results fit the outcomes of a research on medical students' narratives on professionalism dilemmas (Monrouxe and Rees 2012). It is well known that medical students can experience moral distress during their internships; they witness and participate in morally difficult situations, feel responsible and know what would be the right thing to do, but cannot always act as such because of institutional or hierarchical constraints (Monrouxe and Rees 2012; Wiggleton et al. 2010). The types of professionalism dilemmas that evoked the strongest negative emotions were consent dilemmas, patient safety and dignity breaches (by health care professionals) and abuse dilemmas (Monrouxe and Rees 2012). The cases that ethics teachers in our study considered alarming fall within these categories. This seems to imply that the teachers are alarmed by the same kind of cases as the ones that evoke moral distress in students.

\section{Responding to an alarming case within class setting}

The majority of ethics teachers wanted to guard a neutral position as long as possible, because they felt their primary task was to create a safe space for students in which they can express their ideas and feelings freely. Nevertheless, most teachers described a point where the non-judgmental position was given up.

"When it is a black area, I think we (as ethics teachers) should be clear about that" (Interview 14)

From there on, some teachers used the alarming case as a means to reflect with students on their responsibilities and empower them by discussing ways to give feedback in a hierarchical system.

"They have to learn to address things as a clerk: if you don't do it then, you will not do it later. We learn students how to address things properly with the wrongdoer. " (Interview 8)

In some cases teachers advised students to talk to their mentor or supervisor and discuss the case there, or to talk about it with peers. Occasionally, teachers approached a student after class and offered extra support. They would give students their emailaddress for any additional guidance or questions, or contact them afterwards and inform how things went. Teachers agreed that students should never be forced to take action. 
'It is up to the intern to take action or not. Because you go into a procedure in which you are very vulnerable. The perpetrator, let's call it that, has the power, decides on your grade. And doing this anonymously is almost impossible. The perpetrator will find out who has reported it. So that is a very vulnerable position. And so I can understand why someone chooses to stay silent. " (Interview 18)

Literature on medical students' professionalism dilemmas indeed shows that medical students often avoid to speak up for fear of reprisal, of jeopardizing their future career, but also because they feel they lack necessary clinical knowledge and judgment, fear negative consequences on their grades and evaluations or feel they need to be a team player instead of 'rocking the boat' (Christakis and Feudtner 1993; Caldicott and FaberLangendoen 2005; Wiggleton et al. 2010).

\section{Reasons for not intervening outside the scope of the classroom}

Even though the teachers' responses described in the above section can have an effect outside the scope of the classroom, the initiative for taking action was still placed in the hands of the student. The teacher was there to support the student. But did teachers ever consider taking action themselves, outside the scope of the classroom? In other words, did teachers ever feel it was necessary or appropriate to actually intervene themselves? In this study, 12 out of 15 teachers were not eager to take action outside the scope of the classroom (that is, to intervene). This is a remarkable difference with the results of our earlier study on teachers' views in Indonesia, where the majority supported the idea of intervening (Muhaimin et al. 2021). In this section we present the main considerations Dutch teachers gave us for not intervening any further.

\section{A. Ethics class should remain a safe place}

As said before, the majority of our interviewees considered it an important part of their job to create a safe space for students to share and discuss their experiences. Of course this is related to the fears that students have for speaking up, as described in the last paragraph.

'I can tell that they [the students] struggle a lot with this: that they see things and think ' this is not right', but choose to not tell because they still need to be evaluated. And I think it is great that they bring these issues to our ethics class, but then there needs to be a guarantee that their story will stay within the walls of the classroom and that the teacher cannot jeopardize students' position by going about and say: 'yeah well, we heard this and that about your department from an intern.' '(Interview 17)

\section{B. We are not the moral police}

There was a strong belief among our interviewees that ethicists and ethics teachers should be there to help doctors, not tell them what to do. The idea of taking action outside the classroom was often equated with morally judging. 
"We don't focus on who did something wrong, we focus on what is the issue and how you should deal with it properly. We don't take action, no." (Interview 8)

"We are not the safeguard of the hospital. We are not the ethics police!'(Interview 11)

Some of the teachers also worked as an ethical moderator in the hospital. For them, this neutral position is closely linked to their training as moderator, often grounded in hermeneutics:

"My responsibility concerns the deliberation itself, not the situation discussed. This is also what I learned in my training as a moderator: to not participate, but to facilitate."(Interview 18)

The ethics teacher, hence, should not be a moralist, pointing fingers. On the contrary, the ethicist knows that it is often far from obvious what is the right thing to do. Two interviewees explained their aversion against judging, and intervening, as a reaction against the meddling of Protestant-Christian ethicists in debates on medical issues in the 70 's and $80^{\prime}$ in the Netherlands.

"I resist the idea that an ethicist is a sort of secular pastor who tells you what is the right thing to do" (Interview 18)

\section{The ethics dance}

The neutral position of the ethicists, facilitating and supporting doctors, was thus valued and emphasized by almost all our research participants. At the same time, this conviction was sometimes entangled with pragmatic considerations related to the position of ethicists in the hospital.

"Our teaching is embedded in this context of consultancy on one hand and ethics education on the other. We want to help the doctors, to do better or to think. This is a dangerous balance. Because you want to help them to improve, you don't want to say...., they should not feel that we are coming to tell them that what they do is wrong, because the next time they don't come anymore."(Interview 11)

"If we do not guard our neutrality we undermine our own position, become broken-winged." (Interview 18)

"[As an ethics teacher] you have to dance this dance of co-insider and critical outsider and say: "Yes, I am with you. We understand you being a doctor, it's hard work and you try to do the best within limitation.", but you also tell them: "Okay, come out of this medical tunnel vision, and the idea that medicine is all about expertise and rationalism. Be realistic"... 'That's the dance we have to dance.' (Interview 5) 
D. Not our responsibility

The majority of the interviewees felt that it was other people's responsibility to address, report or intervene with alarming cases. In relation to the quality of care, and the wellbeing of patients, for example, responsibility was placed at the (future) care-givers.

"I don't want to know what doctor it was. I don't want that responsibility. It's you as future doctors who should consider taking action. That is not up to me" (Interview 5)

Teachers told us that when students struggle with cases, emotionally or morally, there are other people available for supporting them, such as confidential counselors or mentors. Teachers also encourage students to inform the coordinator of (master) education, as (s)he is considered ultimately responsible for the quality of education during the internships. Ethics teachers generally feel that they have a minor role in the training trajectory of students. Because of the large amount of students and tightly packed medical curriculum, many ethics teachers see students only once or twice during their whole training. Ethics education has a volatile character, "we come in and go again."

"Is it up to me, to judge this situation, and ring the alarm bell, based on 1 moral deliberation-class? Or is it up to people who guide these students more closely, such as tutors, mentors or counselors? Is this my responsibility? And then I eventually decided 'no'." (Interview 19)

\section{E. It is not do-able/ we are not knowledgeable}

The above point is also connected to another aspect of ethics teaching. To protect the confidentiality of patient information teachers are often not informed about the exact location [department] where the alarming case supposedly took place; cases are anonymized. The case may even concern another care institutions or hospital, as interns often work outside the academic hospital.

"I never had the feeling I could do something about it, because the cases are depersonalized. I am not sure which hospital it is and I don't even know most of the interns. It is not doable to act on that."(Interview 13)

Another important, related factor mentioned by interviewees is that ethics teachers only hear half of the story. The student's versions of a case is often somewhat different than the version of the physician or care-givers on site. Ethics teachers, often not medically trained, feel they have to be very careful with handling this type of information.

"Sometimes there is a clinician present in ethics class and they might know the case and add all kinds of relevant nuances, like 'no of course we talked with the family about this', or 'we tried really hard to figure out this alternative route', 
etcetera. Students can be quite blunt in how they describe a certain case, so that made me hesitant in responding too indignant." (Interview 17)

Two more reasons were regularly mentioned by interviewees. First of all, the majority of the ethics teachers simply did not know where to go if they did want to address an alarming issue. In most ethics department there was no clear cut route to deal with such issues or our interviewees did not know of them. Lastly, some teachers referred to their personality as the main reason for not taking action: "I am not the activist type" (Interview 2).

\section{Considerations and circumstances in favor of intervening}

Despite the overall reluctance, teachers also articulated ideological considerations in favor of intervening.

Some teachers told us that the vulnerable position of medical students in the hierarchy of medical education should actually be an incentive to do take action, and not to remain silent (and guard a safe space at all times).

"I think the best way to intervene, normally, is to contact the senior doctor directly. But I think that is not fair to students, because students will not be in a position to say to a senior doctor "well I heard that your behavior is less than ideal'. So then it should be on me." (interview 12)

Others told us why they feel an ethics teacher in particular has a moral responsibility to do something when she hears about an alarming case.

"Part of it is that we show students that we take them serious and that ethical problems are serious problems. To teach them that ethics is not just a nice discussion without consequences... I expect students to behave in an ethical, or at least well-thought-manner, so I should do that myself as well..., like a sort of, in this aspect, a role model. (Interview 14)

There were also practical circumstances that could lead to or support a decision to intervene. When a case regularly popped up in stories of interns, pointing to structural problems at a certain department, the need to intervene increased for teachers. Sometimes teachers approached a head of department, or physician, directly because they happened to know him/her personally. These personal networks often played a role in one's possibilities for taking action. Teachers also remarked that it is helpful to have a background as a physician, or the status of a professor, when addressing an issue.In one department there seemed to be a certain routine for dealing with alarming cases as a team:

"I try to empower them (the students). When that doesn't work, or they are scared, we talk about it in the team, and sometimes, really sometimes, we decide to do something. And that can be anything, can be that we are going to talk to the specialist who supposedly did something wrong, or was hiding something, or we try to let it come out in the open without pointing fingers. 
That depends. I think most of the times, somehow, we find a way out. " (interview 11)

The existence of such a routine of intercollegiate consultation seemed helpful to create the support and possibilities, at least for considering taking action.

Lastly, we noticed that teachers' perspectives on the idea of intervening could change during te interview. By thinking more explicitly end lengthy about these alarming cases and how to respond to them, some teachers started to consider the option of taking action more seriously. Normally, after having felt shocked or overwhelmed by a case during class, they had been taken over by everyday worries soon afterwards and left the case as it was. During the interview they were given an opportunity to reflect more extensively on their experiences and became aware that many more ethics teachers come across such cases- making the cases change from an incident to a more structural (problematic) part of the job. So during the interviews the urge to reflect with colleagues more regularly about this could increase.

" Your research question made me think: ' Should we not so something about this?'. Because everyone in this department does have the experience of reading a case and thinking "How is this even possible?". So I discussed it [the research question] with my colleagues and we were thinking that maybe we should put it on the agenda of next departmental meeting. Because we do not do anything about this now, but shouldn't we? We don't have a shared vision on this, like ' we should intervene', or ' we should not, because this is not our task'. We have all been dealing with this on or own.' (Interview 17)

\section{Actions taken outside class}

To conclude the results section, we present an overview of actual actions taken outside of the classroom (Table 3). There was a certain chronology in the steps taken. First the case was discussed informally with a colleague. After that, in some departments teachers of the ethics department discussed the case together more formally, to decide whether action is appropriate and what that would look like. And only after that, 'real' interventions were done.

Teachers continuously stressed that taking action should be done carefully, not only because of their own position, but even more so because of the doctors'. Things that had to be taken into account when actually approaching the physician or department

Table 3 interventions mentioned by our interviewees

\footnotetext{
Having a moral deliberation with colleagues about the case and what to do

Approaching the doctor/head of department directly (often depends on personal network of the teacher or their background/status as a physician/professor)

Organizing an 'ethics evening' with the ethics team at the department concerned and discussing a similar, anonymized case

Going to a confidential counsellor as an ethics group and reporting the case there
} 
concerned: inform yourself well beforehand about the case, if possible: address the issue directly with the physician/or head of department concerned (never behind someone's back), otherwise bring the issue forward without finger-pointing.

These results confirm the findings of Mannion and Davies in their research on the role of whistleblowing in health organizations (2015). They object to the idea, often found in policy prescriptions that the decision to 'blow the whistle' is a simple matter of individual choice between either speaking up or staying silent. Their research shows that "Before coming to any decision on whether to blow the whistle, employees usually find themselves trying to work out exactly what is happening, often through engaging in dialogue with colleagues and seeking a 'second opinion. "Also, they often look for more informal ways of addressing the issue before, or instead of, blowing the whistle formally (Mannion and Davies 2015).

\section{Discussion}

Literature on the ethical dilemmas of medical students has shown that the professional norms taught during the bachelors phase, including being truthful, respecting patients and colleagues, putting the safety and care of patients first, can differ from the actual behavior and situations they witness during the internships (Christakis and Feudtner 1993; Caldicott and Faber-Langendoen 2005; Wiggleton et al. 2010). This can also be seen in the type of cases that our teachers considered alarming. Or as one of our respondents said:

"The biggest problem is that we teach an ethics that is not completely consonant with reality of medical practice. In year three I've taught them all and they lose a lot of their insights during the internships..... So our biggest challenge [as ethics teachers] is retainment." (interview 5)

As a result, many medical students struggle with their moral integrity during the internships (Wiggleton et al. 2010; Berger 2013; Hamric 2012). Monrouxe and Rees (2012) state that students' predicament is partly due to a culture clash between the older and younger generation physicians. "By teaching them new norms, we send our students to the frontline of cultural change. It is our moral duty [as ethics teachers] to support them in this task." (Monrouxe and Rees 2012). In the setting of an ethics class teachers can try to help students discuss their experiences in a way that relieves tensions and discuss strategies to empower them (for beautiful examples see Berger 2013; Rogers et al. 2012; Gunderson et al. 2009; Gaufberg et al. 2010; Bell and Delbanco 2010).

However, as far as we know, no studies have been done on how ethics teachers perceive their role in this outside the scope of the classroom. Where do they feel their responsibility towards students well-being, and patient care, begin and end? What kind of support can they offer to students who are at the frontline of cultural change? To bring the field of resident ethics further, we wanted more insight in how ethics teachers feel they should and can respond to signals they feel are worrying.

In our study we see that the Dutch ethics teachers we talked to were reluctant to take action outside class. This reluctance is partly rooted in the belief that an ethics teacher 
should stay neutral and facilitate reflection and open exchange of ideas, instead of telling students/doctors what to do. Related to this, an ethics class should be a safe space for students/doctors. This view on teaching seems to be historically grown as well as connected to the role and training of some Dutch ethics teachers as moderators in moral deliberations in hospitals.

Though this neutral and confidential position is highly valued by most teachers, it also seems a hard place to leave for those who would want to or feel they need to. Many interviewed teachers say it would be undoable, or at least very difficult to take action outside the classroom. This has partly to do with the way in which ethics education is organized: the classes are confidential, cases anonymized and there are no long-terms connections between a teacher and a group of students/department. Moreover, many teachers just did not know where to go - there seemed to be no common or well-known route for dealing with alarming cases. This organizational structure hence supports this ideal of the ethicist as being a neutral and facilitating actor.

Some teachers said that the neutral position is a necessary precondition for the ethicist to be able to function in the hospital at all. The ethicist is allowed to support and offer reflection as long as she is not doing so in too much of a threatening way (ref. ethics dance).

This thought links up to some of the considerations we have seen in medical students. They also had a fear of speaking up about medical errors, because they did not want to jeopardize their position. They also wanted to be a team player instead of rocking the boat. And research, in for example the US, UK and Switzerland, has shown that these kinds of considerations can also be found among nurses, allied staff and even among physicians (Nembhard et al. 2015; Schwappach and Gehring 2014). As medical oncologist Srivastava writes: "When I ask colleagues, each recalls sometimes harboring misgivings about another doctor's treatment of a patient but feeling unable or reluctant to comment, even when a patient's life might be threatened -preferring to swallow their discomfort rather than challenge another physician's viewpoint." (Srivastava 2013).

One would expect this so-called silencing culture to come through in our interviews, but it was not addressed as such by the Dutch teachers. Perhaps ethics teachers do not consider themselves part of the organization in this way, also because their responsibility lies more in educating students than in patient care. At the same time, they do work in hospital settings where speaking up is not self-evident and can be considered threatening indeed. It could be an interesting topic to explore further.

When action was taken by ethics teachers, this was done with care and in all examples mentioned we can see serious attempts to guard a safe working environment for both students, teachers and doctors, as well as a safe environment for patients. We also saw that practical circumstances could create more need and/or possibilities to intervene, such as the impact and content of the case, the background and position of the ethics teacher, their personal relational networks, and existing routines of communication about alarming cases within an ethics group. Context matters a lot in these delicate issues, and this might be an indication that ethics teachers indeed prefer to decide on intervening themselves, instead of having to stick to a standard reporting protocol. However, many respondents, also those who said they would never consider 
taking action, told us they would appreciate it if they could share and discuss alarming cases with colleagues more often.

"It would be nice to talk together about these things. I think it is sometimes underestimated, the things we all hear during our job." (Interview 19)

Being neutral and, from there, facilitating open moral reflection, is and will remain a highly valued and anchored position among Dutch ethics teachers. But, based on these results, we do feel that there should also be some possibilities available for teachers to adress an issue or intervene if they feel they need to. Because at this moment the ability to intervene is largely dependent on rather arbitrary circumstances, such as personal networks, strong individual beliefs or professional background. Looking at the needs expressed by our interviewees, a first step could be to discuss alarming cases with colleagues more regularly. By sharing such cases, teachers can stay better connected and knowledgeable of the learning environment of interns and respond, preferable as a group and/or anonymously, when deemed necessary. We agree with Ciasullo et al. (2017), that addressing unjust situations, that are harmful for patients or students, "should be understood as a collective, social, and cultural action rather than an individual initiative". Also, they can explicate together what they think their role as ethics teachers should (and should not) be in the hospital and what responsibilities come with this role. This can strengthen the incentive to actually do take action when needed (see also Henriksen and Dayton 2006).

To conclude: research shows that an important step in relieving the moral stress of interns is to confirm and acknowledge that the norms they learned in class can differ from the way things are done in medical practice. Perhaps the same counts for ethics teachers: If we want to be able to support students in the frontline of cultural change we have to start to acknowledge the ways in which we, as ethics teachers, are (un)able to affirm or challenge existing norms in medical practice and together explore how we might want to proceed from there.

\section{Limitations and further research}

There are some limitations to our study. Firstly, the interviews were done by researchers who are ethics teachers themselves. Even though this means they could relate to the issue well, it could lead to too much of an insiders-situation during the interview as well as during data interpretation, lacking the fresh perspective of an outsider. We did have the advantage of having a mixed team with researchers from Indonesia as well as the Netherlands, which assured that interview data and interpretation were not selfevident to researchers and had to be discussed. Secondly, we only looked at cases that were brought in by students in ethics class. Students sometimes approach an ethics department outside the educational setting, to ask for help or support with a case that they feel is extremely worrying. In these situations, there is a direct appeal from the student to the ethics department (not so much the ethics teacher) to help and/or to intervene. We wanted to limit our study to settings in which there is no explicit appeal, to find out what ethics teachers' considerations are and to keep the research question as clear as possible. But by doing so, we had to block out cases that could have been 
informative on what routes are available for ethicists in the hospital to address i.e. misconduct.

Thirdly, the focus on neutrality in ethics teaching and moral deliberation might be typical for the Dutch setting; we have seen that in Indonesia this is not a central topic for teachers (Muhaimin et al. 2021). We do believe, however that the question of intervening or not can be a complicated matter for ethics teachers in many more countries. And that it is important for ethics teachers anywhere in the world to explore the (im)possibilities of addressing unjust situations from their specific positions in medical institutions.

This study does not reveal what other groups working and studying in the hospital think about the responsibilities and task of ethics teachers, such as medical students, physicians, mentors and managers, in relation to alarming cases. This would be an interesting topic to research further. We also think it would be valuable to take a next step and invite Dutch ethics teachers to sit together and exchange ideas, i.e. in a focus group, on what could or could not be appropriate routes for addressing alarming cases in a hospital setting as an ethics department.

Acknowledgments We would like to thank all interviewees for generously sharing their thoughts and experiences and for reading and commenting on the manuscript before publication. We would also like to thank Raditya Bagas Wicaksono for helping us out with the interviews and transcribing, and our colleagues Boukje van der Zee and Wieke Ligtenberg for giving feedback on earlier drafts.

Availability of data and material (data transparency) because of confidentiality agreements with the interviewees, materials are only available on request.

Code availability Not applicable.

Funding This research was made possible by the Institute for Education (IOO: Instituut voor Onderwijs en Opleiden) of the Amsterdam University Medical Center. This study is also part of a larger study funded by the Ministry of Research, Technology and Higher Education of the Republic of Indonesia for the PhD project carried out by Amalia Muhaimin, award number 238/D3.2/PG/2016.

\section{Declarations}

Ethical approval This study did not have any direct contact or interventions with patients, and therefore was not required to apply for ethical approval from the research ethics committees of the Amsterdam UMC.

Consent to participate Interviewees were asked written permission to participate in the research after having been informed on the exact interview procedure.

Consent for publication Interviewees were asked written permission for the researchers to publish results of this research.

Conflicts of interest/competing interests On behalf of the authors, the corresponding author states that there is no conflict of interest.

Open Access This article is licensed under a Creative Commons Attribution 4.0 International License, which permits use, sharing, adaptation, distribution and reproduction in any medium or format, as long as you give appropriate credit to the original author(s) and the source, provide a link to the Creative Commons licence, and indicate if changes were made. The images or other third party material in this article are included in the 
article's Creative Commons licence, unless indicated otherwise in a credit line to the material. If material is not included in the article's Creative Commons licence and your intended use is not permitted by statutory regulation or exceeds the permitted use, you will need to obtain permission directly from the copyright holder. To view a copy of this licence, visit http://creativecommons.org/licenses/by/4.0/.

\section{References}

Bell, S.K., and T. Delbanco. 2010. Improving the patient, family, and clinical experience after harmful events: the "When things go wrong"curriculum. Academic Medicine 85: 1010-1017.

Berger, J. T. 2013. 'Moral distress in medical education and training.' Journal of General Internal Medicine: 395-398.

Caldicott, Catherine V., and Kathy Faber-Langendoen. 2005. Deception, discrimination, and fear of reprisal: lessons in ethics from third-year medical students. Academic Medicine 80 (9): 866-873.

Christakis, Dimitri A., and Chris Feudtner. 1993. Ethics in a short white coat: the ethical dilemmas that medical students confront. Academic medicine: journal of the Association of American Medical Colleges 68 (4): 249-254.

Ciasullo, Maria Vincenza, Silvia Cosimato, and Rocco Palumbo. 2017. 'Improving health care quality: The implementation of whistleblowing.' The TQM Journal.

Gaufberg, E.H., M. Batalden, R. Sands, and S.K. Bell. 2010. The Hidden Curriculum: What can we learn from third-year medical student narrative reflection? Academic Medicine 85 (11): 1709-1712.

Gunderson, A.J., K.M. Smith, D.B. Mayer, T. McDonald, and N. Centomani. 2009. Teaching medical students the art of medical error full disclosure: evaluation of a new curriculum. Teaching learning medicine 21: 229-232.

Hamric, A.B. 2012. Empricial research on moral distress: issues, challenges, and opportunities. HEC forum 24: $39-49$.

Henriksen, Kerm, and Elizabeth Dayton. 2006. 'Organizational silence and hidden threats to patient safety.' Health services research 41.4p2: 1539-1554.

Kolb, D. A. 2014. Experiential learning: Experience as the source of learning and development. FT press.

Mannion, Russell, and Huw TO Davies. 2015. Cultures of silence and cultures of voice: the role of whistleblowing in healthcare organisations. International journal of health policy and management 4 (8): 503.

Monrouxe, L.V., and C.E.R. Rees. 2012. "It's just a clash of cultures": emotional talk within medical students'narratives of professionalism dilemmas. Advances in Health science Education 17: 671-701.

Muhaimin, A., Willems, DL., Utarini, A. and Maartje H. 2019. What do students perceive as ethical problems? A comparative study of Dutch and Indonesian medical students in clinical training. Asian Bioethics Review 11 (4):391-408.

Muhaimin, A., Maartje, H., Utarini, A. and Willems, D.L. 2020. Ethics education should make room for emotions: a qualitative study of medical ethics teaching in Indonesia and the Netherlands. International Journal of Ethics Education 5 (1): 7-21.

Muhaimin, A., Maartje, H., Wicaksono, R.B., Utarini, A. and Willems, D.L. 2021. "I would do something if I could!": experiences and reflections from ethics teachers on how to respond when hearing alarming cases from medical students. BMC Medical Education, 21(1): 1-11.

Nembhard, I.M., I. Labao, and S. Savage. 2015. Breaking the silence: determinants of voice for quality improvement in hospitals. Health Care Management Review 40 (3): 225-236.

Rees, Charlotte E., and Lynn V. Monrouxe. 2011. Medical students learning intimate examinations without valid consent: a multicentre study. Medical Education 45 (3): 261-272.

Rogers, D.A., M.L.B. Roberts, and V. Johnson. 2012. Using the Hidden Curriculum to Teach Professionalism During the Surgery Clerkship. Journal of Surgical Education 69: 423-427.

Schwappach, David L.B., and Katrin Gehring. 2014. Trade-offs between voice and silence: a qualitative exploration of oncology staff's decisions to speak up about safety concerns. BMC health services research 14 (1): 1-10.

Srivastava, Ranjana. 2013. Speaking up —when doctors navigate medical hierarchy. New England Journal of Medicine 368 (4): 302-305. 
Wiggleton, C., E. Petrusa, K. Loomis, J. Tarpley, M. Tarpley, M.L. O’Gorman, and B. Miller. 2010. Medical students' experience of moral distress: development of a web-based survey. Academic Medicine 85 (1): 111-117.

Publisher's note Springer Nature remains neutral with regard to jurisdictional claims in published maps and institutional affiliations.

\section{Affiliations}

\section{Maartje Hoogsteyns ${ }^{1} \cdot$ Amalia Muhaimin $^{1,2}$}

1 Department of Ethics, Law and Medical Humanities, Amsterdam UMC, Amsterdam, The Netherlands

2 Department of Bioethics and Humanities, Faculty of Medicine, Universitas Jenderal Soedirman, Kampus Kedokteran, Jl. Dr. Gumbreg 1, Purwokerto, Indonesia 